\title{
Optimization and stabilization of sequential learning in RBF network for nonlinear function approximation
}

\author{
W. S. Lim ${ }^{\mathrm{a})}$ and J. W. L. Yeoh \\ Faculty of Engineering \& Technology, Multimedia University, \\ Jalan Ayer Keroh Lama, 75450 Melaka, Malaysia \\ a)wslim@mmu.edu.my
}

Abstract: This paper proposes a solution for inconsistency pruning of neurons within a sequential learning Radial Basis Function (RBF) Network. This paper adopts the concept that a specific RBF neuron which continuously exhibits low output in a sequence of training patterns does not justify the proposition that the neuron is insignificant to the whole function to be approximated. We establish additional criterions to provide protection from error in pruning RBF neurons within the hidden layer, which we prove is able to improve consistency and stability of neuron evolution. With such stability within the sequential learning process, we also show how the convergence speed of the network can be improved by reducing the number of consecutive observations required to prune a neuron in the hidden layer.

Keywords: feedforward neural networks, Radial Basis Network $(\mathrm{RBF})$, sequential learning, nonlinear function approximation, minimal resource allocation network

Classification: Science and engineering for electronics

\section{References}

[1] N. Sundararajan, P. Saratchndran, and Y. W. Lu, "Radial Basis Function Neural Networks with Sequential Learning," World Scientific, Singapore, 1999.

[2] Y. W. Lu, N. Sundararajan, and P. Saratchandran, "A sequential learning scheme for function approximation using minimal radial basis function neural networks," Neural Comput., vol. 9, pp. 461-478, 1997.

[3] J. P. Deng, N. Sundararajan, and P. Saratchandran, "Communication Channel Equalization Using Complex-Valued Minimal Radial Basis Function Neural Networks," IEEE Trans. Neural Networks, vol. 13, pp. 687696, 2002.

[4] Y. W. Lu, N. Sundararajan, and P. Saratchandran, "Performance evaluation of a sequential minimal Radial Basis Function (RBF) neural network learning algorithm," IEEE Trans. Neural Networks, vol. 9, pp. 308-318, 1998. 
[5] G. B. Huang, P. Saratchandran, and N. Sundararajan, "A generalized growing and pruning RBF neural network for function approximation," IEEE Trans. Neural Networks, vol. 16, no. 1, pp. 57-67, 2005.

[6] V. Kadirkamanathan and M. Niranjan, "A function estimation approach to sequential learning with neural networks," Neural Comput., vol. 5, pp. 954-975, 1993.

\section{Introduction}

To address the need for a simple and practical implementation of neural networks with optimized computation overhead, a branch of RBF based neural network utilizing sequential learning called the Minimal Resource Allocation Network (MRAN) has been developed by Lu et al. [1, 2]. The MRAN algorithm manages the size of the neural network dynamically by utilizing specific algorithms for adding and pruning RBF hidden neurons as necessary, so that an optimized implementation of the RBF network can be attained as a result of supervised training.

Ever since the pruning algorithm in MRAN was introduced, the MRAN algorithm has been successfully applied and furthermore, improvised to attain optimal neuron configurations in the hidden layer as in EMRAN and HMRAN developed by $\mathrm{Li}$ et al. and Nishida et al. in $[3,4]$. By removing unnecessary neurons in the hidden layer of the network, minimal computation power is required in the actual implementation of the system. The algorithm used in the original algorithm of MRAN decides whether a neuron should be pruned by taking into account the normalized absolute output of that neuron with respect to the absolute output of the largest contributing neuron. If a specific neuron does not contribute any significant amount of output over a certain number of training iterations, the neuron will be pruned.

Success has been achieved in implementations of MRAN on communication channel equalization by Deng et al. [5] employing an extended learning algorithm known as Complex MRAN (CMRAN). MRAN has also been successful applied to solve problems in areas of function approximation and time series prediction in $[1,2]$. However, as will be shown later in this paper, there is a huge inconsistency issue when MRAN is used to solve problems in the area of function approximation. The nature of the data set used in supervised training of the network can occasionally cause the pruning mechanism used in MRAN to prune neurons in the hidden layer that contribute significantly to the function being approximated. This mistake in pruning happens when the data set does not oscillate through all possible input combination consistently and uniformly enough to maintain relevance of each RBF neuron. Therefore, it is important we acknowledge that a specific neuron which is not used in a sequence of training patterns does not justify that the neuron is insignificant to the whole function to be approximated. Because of this, we need to establish additional criterions to the pruning process so that these pruning inconsistencies can be eliminated. 


\section{Improved Pruning Criterion}

In this section, we propose additional criterions to be added into the pruning process. We first refresh ourselves on the types of neurons that we are interested in pruning from the hidden layer of the artificial neural network. Unwanted neurons within the network are either neurons which does not contribute to approximating any part of the desired function or neurons which contribute to the negative performance of a system. Quite obviously, a neuron which does not contribute to approximating any part of the desired function can only be a neuron which gives a low output to all output nodes regardless of the input vector being introduced to the neuron, giving rise to inequality (1) as an additional pruning criterion.

$$
\mathrm{w}_{\mathrm{i}}<\mathrm{k}\left(\mathrm{Y}_{\mathrm{MAX}}-\mathrm{Y}_{\mathrm{MIN}}\right)
$$

where $\mathrm{w}_{\mathrm{i}}$ is the weight of hidden $\mathrm{i}^{\text {th }}$ neuron, and $\mathrm{k}$ is the significance threshold which determines the amount of neuron contribution that is needed to consider the neuron a significant neuron. In typical conditions, a value between 0.01 and 0.05 is used for the value of $\mathrm{k}$. However, it should also be noted that the value of $\mathrm{k}$ affects the maximum desired accuracy of the system response. $\mathrm{Y}_{\mathrm{MAX}}$ and $\mathrm{Y}_{\mathrm{MIN}}$ denote the maximum and minimum output of the training pattern provided respectively. This inequality can be extended to networks with more than one output by comparing the maximum weight of a particular neuron in the network with its corresponding maximum and minimum training output as in inequality (2).

$$
\max \left(\mathrm{w}_{\mathrm{i}}{ }^{1}, \mathrm{w}_{\mathrm{i}}{ }^{2}, \mathrm{w}_{\mathrm{i}}{ }^{3}, \ldots \mathrm{w}_{\mathrm{i}}^{\mathrm{p}}\right)<\mathrm{k}\left(\mathrm{Y}_{\mathrm{MAX}}{ }^{\mathrm{j}}-\mathrm{Y}_{\mathrm{MIN}^{\mathrm{j}}}{ }^{\mathrm{j}}\right)
$$

where $\mathrm{Y}_{\mathrm{MAX}}{ }^{\mathrm{j}}$ and $\mathrm{Y}_{\mathrm{MIN}}{ }^{\mathrm{j}}$ are the maximum and minimum expected output presented by the training data for output node $\mathrm{j}$, output node $\mathrm{j}$ is the output node having the highest weight from $\mathrm{i}^{\text {th }}$ neuron, and $\mathrm{p}$ is the number of output nodes in the network. These inequalities compare the highest weight of a neuron with a fraction of the expected output range. However, this does not mean that neurons responsible to model small amplitude portions of the function will become more likely to be eliminated. As in the original version of pruning algorithm, pruning of neurons is still done conditionally, depending on the normalized contribution of each neuron. The weight criterion merely acts as a countermeasure to prevent any mistake in pruning neurons within the hidden layer.

Once this criterion has been implemented successfully, we will notice the existence of another type of neuron that should and would have been pruned with the original pruning algorithm but failed to be pruned with the introduction of the new criterion.

In RBF neural networks, neuron parameters are adjusted to find the best fit for a neuron. Neurons in RBF networks can be grown by mistake due to noise in the training data set or neurons made obsolete in a dynamic system. The undesired neuron will then be handled by either reducing the weight of the Gaussian activation function, reducing the variance of the Gaussian 
function, moving the center of the Gaussian function within the neuron or a combination of a few or all of these solutions, depending on the learning algorithm being used. Consequently, there exists a type of undesired neuron having an extremely small Gaussian activation function variance, but high enough output weight so that it gets itself excluded from pruning.

With the purpose of eliminating this inconsistency in pruning, an additional criterion as in inequality (3) will be added to the pruning process.

$$
\sigma_{\mathrm{i}}<\mathrm{T}
$$

where $\sigma_{i}$ denotes the variance of $\mathrm{i}^{\text {th }}$ neuron, and $\mathrm{T}$ is the threshold of which we will subject any neurons with a variance of less than such value to possible pruning. Because this criterion is only meant to remove these extremities, the threshold $\mathrm{T}$ should be selected to be around 0.01 of the input domain.

Bringing the criterions to act together as one will result in the antecedent as in condition (4).

$$
\mathrm{r}_{\mathrm{ji}}{ }^{\mathrm{n}}<\delta \text { AND }\left(\mathrm{w}_{\mathrm{i}}^{\mathrm{j}}<\mathrm{k}\left(\mathrm{Y}_{\mathrm{MAX}}^{\mathrm{j}}-\mathrm{Y}_{\mathrm{MIN}}{ }^{\mathrm{j}}\right) \text { OR } \sigma_{\mathrm{i}}<\mathrm{T}\right)
$$

where $\mathrm{r}_{\mathrm{ji}}{ }^{\mathrm{n}}$ is the normalized absolute output of $\mathrm{i}^{\text {th }}$ neuron at $\mathrm{n}^{\text {th }}$ iteration to output node $\mathrm{j}$, and $\delta$ is the output significance threshold.

\section{Simulation Results and Discussion}

Proposal to include the two additional criterions for pruning in the fashion as described in the earlier section has been assessed by means of simulation using MATLAB. Our objective in this test is to show the circumstances in which error in pruning can be avoided and how we can further improve the pruning efficiency of the network. This experiment will not attempt to train the neural network in the least number of training patterns, but will instead attempt to demonstrate how the improved pruning criterions will relatively increase the stability of the network and the speed of convergence during supervised training. We initially test the new criterions on a nonlinear function that was used to previously to evaluate a new hybrid learning scheme for Gaussian potential function networks [1] and to compare the original MRAN algorithm with RANEKF [6]. The nonlinear function is a combination of six Gaussian functions given as in Equation (5).

$$
\begin{aligned}
y(x) & =\exp \left\{-\left[\frac{\left(x_{1}-0.3\right)^{2}+\left(x_{2}-0.2\right)^{2}}{0.01}\right]\right\}+\exp \left\{-\left[\frac{\left(x_{1}-0.7\right)^{2}+\left(x_{2}-0.2\right)^{2}}{0.01}\right]\right\} \\
& +\exp \left\{-\left[\frac{\left(x_{1}-0.1\right)^{2}+\left(x_{2}-0.5\right)^{2}}{0.01}\right]\right\}+\exp \left\{-\left[\frac{\left(x_{1}-0.9\right)^{2}+\left(x_{2}-0.5\right)^{2}}{0.01}\right]\right\} \\
& +\exp \left\{-\left[\frac{\left(x_{1}-0.3\right)^{2}+\left(x_{2}-0.8\right)^{2}}{0.01}\right]\right\}+\exp \left\{-\left[\frac{\left(x_{1}-0.7\right)^{2}+\left(x_{2}-0.8\right)^{2}}{0.01}\right]\right\}
\end{aligned}
$$

In this simulation, we utilized a dataset of 5000 instances which will be fed sequentially to the network during supervised training. The number of 


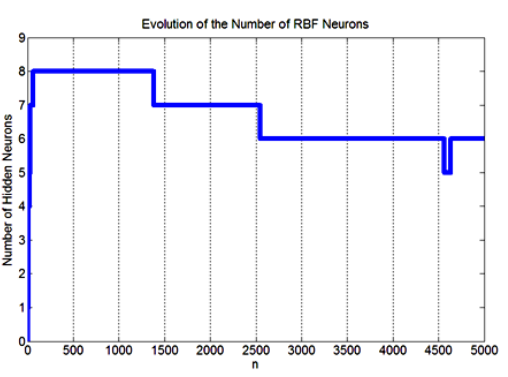

(a)

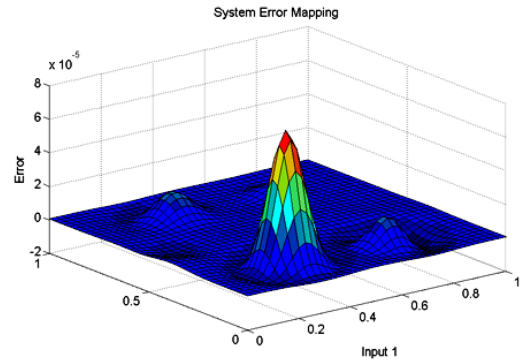

(c)

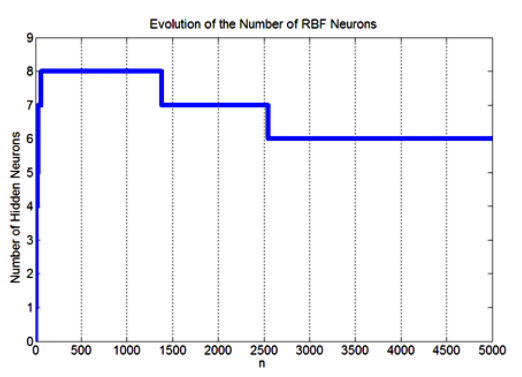

(b)

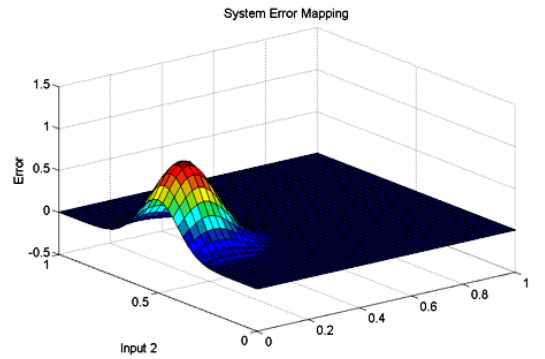

(d)

Fig. 1. (a) Evolution of the number of RBF neurons in a network with unmodified criterions. (b) Evolution of the number of RBF neurons in a network with proposed criterions added. (c) Error mapping of the network with unmodified criterions at iteration 4562. (d) Error mapping of the network with unmodified criterions at iteration 4563 .

neurons within the hidden layer was monitored and the effect of each neuron pruning onto the network response was analyzed.

As we can see in the case of the unmodified criterion (Figure $1(\mathrm{a})$ ), the convergence of the number of neurons towards the optimal solution was erroneously disturbed by the pruning mechanism at iteration 4562 . Figures 1 (c), (d) shows the error mapping of the RBF network with respect to the input space at iterations 4562 and 4563 respectively. In the former plot, six neurons were used to model Equation (5), which gives an excellent approximation with a maximum error of $7.9177 \times 10^{-5}$. When the network progresses into the next iteration, one of its six critical neurons was erroneously pruned resulting in a maximum error of 1 which is the amplitude of Gaussian functions inside the function to be approximated (as in Figure 1(d)).

Intuitively, this problem can be solved by increasing the consecutive observations of low neuron contribution required for the pruning algorithm to make a decision to prune the neuron in the hidden layer. We call this value the insignificant time limit of the algorithm, denote it by $\mathrm{n}_{\mathrm{w}}$ and increase this variable from 30 to 50 in order to eliminate such occurrences of mispruning. However, this is a bad solution to the pruning problem, particularly in function approximation problems. There exist two reasons for this: i) increasing the amount of iterations needed to prune a neuron would also mean that the system will require more number of iterations to converge upon the correct approximation; ii) the problem is not eliminated completely, only that the 


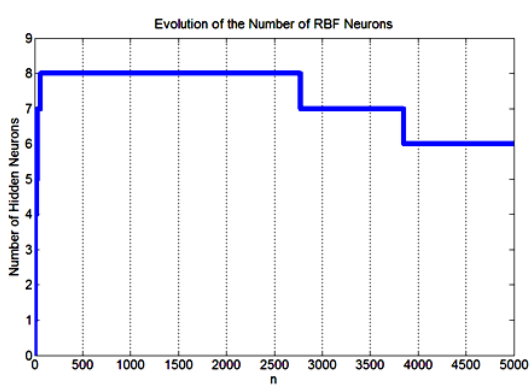

(a)

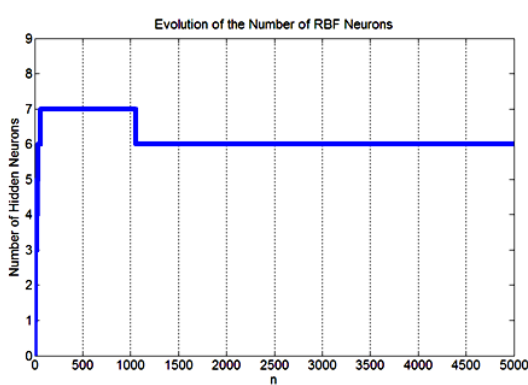

(b)

Fig. 2. (a) Evolution of number of RBF neurons with unmodified criterions using $\mathrm{n}_{\mathrm{w}}=50$. (b) Evolution of number of RBF neurons with modified criterions using $\mathrm{n}_{\mathrm{w}}=1$.

probability of erroneous pruning is lessened.

Figure 2 shows successful convergence of two different RBF networks to the optimal number of six neurons to approximate Equation (5), one of which is the unmodified version of the algorithm, while another has been trained using the improved pruning criterions. The insignificant time limit of the unmodified version of the algorithm $\mathrm{n}_{\mathrm{w}}$ has been set to 50 to prevent erroneous pruning in training. On the other hand, $\mathrm{n}_{\mathrm{w}}$ in the improved algorithm has been reduced to 1 to improve pruning efficiency. We can observe that the former network takes nearly 4000 iterations to successfully achieve the optimal number of neurons while the improved algorithm only requires about 1000 iterations, demonstrating a highly increased speed of convergence.

The algorithm has also been tested on functions of different nature, including linear functions, step functions as well as multidimensional sinusoidal functions. The improvement has shown reliable ability to converge to a stable state, effectively approximating those functions.

\section{Conclusion}

In this paper, the algorithm for RBF neuron growth and pruning has been made specialized for function approximation, eliminating a major consistency and stability issue in RBF neuron evolution, as well as greatly increasing the speed of convergence in MRAN. Two additional criterions have been added to the existing algorithm, in order to prevent accidental removal of RBF neurons essential to modeling the desired function. A neuron would have to exhibit relatively low Gaussian function weight with respect to the function output, or a small Gaussian activation function variance, in order to qualify itself for pruning. Elimination of such inconsistencies allows us to heavily reduce the number of consecutive observations needed to prune neurons. In effect, more iteration can be used to further tweak the fine details of the function to be approximated. From the simulation results, elimination of error in pruning and further increase in speed of convergence is authenticated. 